\title{
SIGMOIDAL COSINE SERIES ON THE INTERVAL
}

\author{
BEONG IN YUN ${ }^{1}$
}

(Received 17 February, 2005)

\begin{abstract}
We construct a set of functions, say, $\psi_{n}^{[r]}$ composed of a cosine function and a sigmoidal transformation $\gamma_{r}$ of order $r>0$. The present functions are orthonormal with respect to a proper weight function on the interval $[-1,1]$. It is proven that if a function $f$ is continuous and piecewise smooth on $[-1,1]$ then its series expansion based on $\psi_{n}^{|r|}$ converges uniformly to $f$ so long as the order of the sigmoidal transformation employed is $0<r \leq 1$. Owing to the variational feature of $\psi_{n}^{|r|}$ according to the value of $r$, one can expect improvement of the traditional Fourier series approximation for a function on a finite interval. Several numerical examples show the efficiency of the present series expansion in comparison with the Fourier series expansion.
\end{abstract}

2000 Mathematics subject classification: primary 41 A58; secondary 42A20.

Keywords and phrases: Fourier series, sigmoidal transformation, sigmoidal cosine series.

\section{Introduction}

The sigmoidal transformation is a one-to-one mapping of the interval $[0,1]$ onto itself taking the shape of an elongated " $\mathrm{S}$ ". It is well known that coordinate transformation techniques using a sigmoidal transformation are very efficient for accurate numerical evaluation of singular integrals such as weakly singular integrals, Cauchy principal value integrals and Hadamard finite-part integrals [1-4, 8, 11-14].

In this paper, as an extended application of the sigmoidal transformation, we construct a trigonometric function denoted by $\psi_{n}^{[r]}(x),-1 \leq x \leq 1$, which is composed of a cosine function and a sigmoidal transformation $\gamma_{r}(t), 0 \leq t \leq 1$, of order $r>0$. Owing to the particular behaviour of $\gamma_{r}$, the function $\psi_{n}^{[r]}$ has the property that its zeros are clustered toward both endpoints of the interval $[-1,1]$ for small $r<1$ and the centre for large $r>1$. Moreover, it can be seen that for any $r>0$ the set $\left\{\psi_{n}^{(r)}\right\}_{n=0}^{\infty}$ is

\footnotetext{
'Faculiy of Mathematics, Informatics and Statistics, Kunsan National University, 573-701, Korea; e-mail: biyun@kunsan,ac.kr or paulll@maths.uq.edu.au.

(C) Australian Mathematical Society 2006, Serial-fee code 1446-1811/06
} 
orthonormal on $[-1,1]$ with respect to a weight function $w^{[r]}$ defined in Section 2. In fact it is an orthonormal basis for the weighted $L_{2}$ space, $L_{2, w}([-1,1])$. When $r=1$, in particular, $w^{[1]} \equiv 1$ and $\left\{\psi_{n}^{[1]}\right\}_{n=0}^{\infty}$ is identical to a simple basis $\left\{\cos \left(k \pi \frac{1+x}{2}\right)\right\}_{n=0}^{\infty}$ for $L_{2}([-1,1])$. This means that according to an arbitrary $r$ we can develop infinitely many orthonormal bases generalising the traditional Fourier cosine functions.

We are mainly interested in the series expansion, say, $S^{[r]}(f ; x)$ of a piecewise smooth function $f$ on $[-1,1]$ based on the present orthonormal set $\left\{\psi_{n}^{(r)}\right\}_{n=0}^{\infty}$. Convergence analysis for the series $S^{[r]}(f ; x)$ tells us that it converges pointwise to an average value of left- and right-side limits $\left\{f\left(x_{-}\right)+f\left(x_{+}\right)\right\} / 2$ for each $x \in(-1,1)$. At the endpoints $x= \pm 1, S^{[r]}(f ; x)$ converges to $f\left(1_{-}\right)=\lim _{x \rightarrow 1-} f(x)$ and $f\left(-1_{+}\right)=\lim _{x \rightarrow-1+} f(x)$, respectively. Moreover if $f$ is continuous as well as piecewise smooth on $[-1,1]$ then for any $0<r \leq 1$ the uniform convergence of the series $S^{[r]}(f ; x)$ to $f(x)$ can be proved. Additionally, results of the pointwise and uniform convergence of the series $S^{[r]}(f ; x)$ are extended to a function $f$ whose derivative has weak singularities at the endpoints of the interval. On the other hand, in order to show the potential of the series $S^{[r]}(f ; x)$, the Gibbs phenomenon for a discontinuous function and the periodic extension are also investigated.

Approximation to a function $f$ by the $N$ th partial sum $S_{N}^{[r]}(f ; x)$ of $S^{[r]}(f ; x)$ is important in practical applications. For instance we consider several functions for which the traditional Fourier series approximation is not satisfactory. From the numerical results we can take notice of the superiority of the present series approximation, at least, for the function having a pick near a centre or endpoints of the interval $[-1,1]$ and for the function whose derivative has weak singularities.

This paper is organised as follows. In Section 2 we define a generalised sigmoidal transformation and then for any $r>0$ construct an orthonormal set of functions $\psi_{k}^{[r]}$, $k=0,1,2, \ldots$, by using the Fourier cosine function and the sigmoidal transformation. In Section 3 we consider a series expansion based on the functions $\psi_{k}^{[r]}$ called a sigmoidal cosine series (SCS). Using the inherent properties of the sigmoidal transformation, we prove the pointwise and uniform convergence of the SCS which forms the main theorems of this paper. In Section 4 the Gibbs phenomenon for a discontinuous function is investigated and Section 5 includes comments on the periodic even extension of a function defined on the half-interval $[0,1]$. Several numerical examples are worked through in Section 6 to show the availability of the SCS compared with the traditional Fourier series.

\section{Construction of orthonormal sets}

We define the sigmoidal transformation, whose original definition can be found in the literature $[3,4]$, as follows. 
DEFINITION 2.1. For any $r>0$, a real-valued function denoted by $\gamma_{r}(y)$ which has the following properties is called a sigmoidal transformation of order $r$ :

(S1) $\gamma_{r}(y) \in \mathbb{C}[0,1] \cap \mathbb{C}^{\infty}(0,1)$ with $\gamma_{1}(y)=y$.

(S2) $\gamma_{r}(y)+\gamma_{r}(1-y)=1,0 \leq y \leq 1$.

(S3) $\gamma_{r}(y)$ is strictly increasing on $[0,1]$ with $\gamma_{r}(0)=0$.

(S4) On the subinterval $[0,1 / 2]$, the first derivative $\gamma_{r}^{\prime}(y)$ is strictly increasing when $r>1$, and it is strictly decreasing when $r<1$.

(S5) Near $y=0, \gamma_{r}^{(j)}(y)=O\left(y^{r-j}\right), j=0,1,2, \ldots,\lfloor r\rfloor$, where $\lfloor r\rfloor$ denotes the greatest integer less than or equal to $r$.

In fact $\gamma_{r}(x)$ is a one-to-one mapping from $[0,1]$ onto itself and its inverse $\gamma_{r}^{-1}$ is strictly increasing on $[0,1]$ and, from (S2), it also satisfies the relation $\gamma_{r}^{-1}(y)+$ $\gamma_{r}^{-1}(1-y)=1$. Further it follows that for all $0 \leq y \leq 1$

$$
\gamma_{r}^{\prime}(y)=\gamma_{r}^{\prime}(1-y), \quad \gamma_{r}^{\prime \prime}(y)+\gamma_{r}^{\prime \prime}(1-y)=0,
$$

and, similarly to (S5), near $y=1$

$$
\gamma_{r}(y)=1+O\left((1-y)^{r}\right)
$$

and

$$
\gamma_{r}^{(j)}(y)=O\left((1-y)^{r-j}\right), \quad j=1,2, \ldots,\lfloor r\rfloor .
$$

In addition it is observed that $\gamma_{r}(1 / 2)=1 / 2$ and $\gamma_{r}^{\prime \prime}(1 / 2)=0$. As an example of a sigmoidal transformation, we introduce a simple sigmoidal transformation [10] such as

$$
\gamma_{r}(y)=\frac{y^{r}}{y^{r}+(1-y)^{r}}, \quad 0 \leq y \leq 1,
$$

which has the particular properties that $\gamma_{r}^{-1}=\gamma_{1 / r}$ and $\gamma_{r}^{\prime}(1 / 2)=r$.

We define new functions such as

$$
\psi_{k}^{[r]}(x):=\cos \left[k \pi \gamma_{r}((1+x) / 2)\right], \quad k=1,2,3, \ldots,
$$

with $\psi_{0}^{[r]}(x):=1 / \sqrt{2}$. Then for a weight function defined as

$$
w^{[r]}(x):=\gamma_{r}^{\prime}((1+x) / 2)>0, \quad-1<x<1,
$$

we have, by a change of variables $t=\gamma_{r}((1+x) / 2)$,

$$
\begin{aligned}
\left\langle\psi_{i}^{[r]}, \psi_{j}^{[r]}\right\rangle_{w} & :=\int_{-1}^{1} w^{[r]}(x) \psi_{i}^{[r]}(x) \psi_{j}^{[r]}(x) d x \\
& =2 C_{i} C_{j} \int_{0}^{1} \cos (i \pi t) \cos (j \pi t) d t=\delta_{i j},
\end{aligned}
$$


where the value of $C_{k}$ is $1 / \sqrt{2}$ when $k=0$, and 1 when $k \neq 0$. That is, the set $\left\{\psi_{k}^{[r]}(x)\right\}_{k=0}^{\infty}$ is orthonormal with respect to the inner product $\langle\cdot, \cdot\rangle_{w}$. Moreover, noting that $\{\cos k \pi t\}_{k=0}^{\infty}$ is an orthonormal basis for $L_{2}([0,1])$, it is straightforward that $\left\{\psi_{k}^{[r]}(x)\right\}_{k=0}^{\infty}$ becomes an orthonormal basis for the weighted $L_{2}$ space, $L_{2, w}([-1,1])$ equipped with a norm $\|f\|_{2, w}=\langle f, f\rangle_{w}^{1 / 2}$.

For a special case of $r=1$ it can be found that since $\gamma_{1}(x)$ is an identity map the function $\psi_{k}^{[1]}(x)$ with $w^{[1]}(x)=1$ produces

$$
\psi_{k}(x):=\psi_{k}^{(1)}(x)=\cos [k \pi(1+x) / 2],
$$

for $k=1,2,3, \ldots$, with $\psi_{0}(x):=1 / \sqrt{2}$. Thus we can note that the present function $\psi_{k}^{[r]}(x)$ is a generalisation of the simple cosine function $\psi_{k}(x)$ accompanying a varied weight function $w^{[r]}(x)$ with respect to $r>0$.

THEOREM 2.1. For each integer $k \geq 1$ the function $\psi_{k}^{[r]}(x)$ with $r>0$ has the following properties:

(i) $\psi_{k}^{[r]}(-1)=1, \psi_{k}^{[r]}(0)=\cos [k \pi / 2], \psi_{k}^{[r]}(1)=(-1)^{k}$;

(ii) $\psi_{k}^{[r]}(-x)=(-1)^{k} \psi_{k}^{[r]}(x)$;

(iii) near $x=-1$ and $x=1$, respectively,

$$
\psi_{k}^{[r]}(x)=1+O\left((1+x)^{2 r}\right), \quad \psi_{k}^{[r]}(x)=(-1)^{k}+O\left((1-x)^{2 r}\right) ; \quad \text { and }
$$

(iv) the zeros of $\psi_{k}^{[r]}$ are

$$
x_{k, j}^{[r]}=2 \gamma_{r}^{-1}\left(\frac{2 j-1}{2 k}\right)-1, \quad j=1,2, \ldots, k,
$$

which satisfy $-1<x_{k, 1}^{[r]}<x_{k, 2}^{[r]}<\cdots<x_{k, k-1}^{[r]}<x_{k, k}^{[r]}<1$ and

$$
x_{k, j}^{[r]}=-x_{k, k-j+1}^{[r]}, \quad j=1,2, \ldots,\lfloor(k+1) / 2\rfloor .
$$

PROOF. Since $\gamma_{r}(0)=0, \gamma_{r}(1 / 2)=1 / 2$ and $\gamma_{r}(1)=1$ property (i) is clear. From (S2) and the definition of $\psi_{k}^{[r]}$ in (2.2),

$$
\psi_{k}^{[r]}(-x)=\cos \left[k \pi\left\{1-\gamma_{r}\left(\frac{1+x}{2}\right)\right\}\right]=(-1)^{k} \cos \left[k \pi \gamma_{r}\left(\frac{1+x}{2}\right)\right],
$$

which proves property (ii). Then property (iii) can be derived by observing the local behaviour of $\gamma_{r}$ given in (S5) and the Taylor series expansion of the cosine function. Finally, solving the straightforward equation $\psi_{k}^{[r]}(x)=0$, we have solutions of the form (2.5). Since the inverse $\gamma_{r}^{-1}$ of $\gamma_{r}$ is also strictly increasing and it satisfies the relation $\gamma_{r}^{-1}(\eta)+\gamma_{r}^{-1}(1-\eta)=1$ on the interval $[0,1]$, the zeros $x_{k, j}^{[r]}$ are strictly increasing and (2.6) holds. 


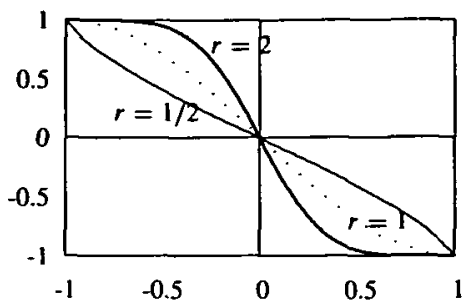

(a) $k=1$

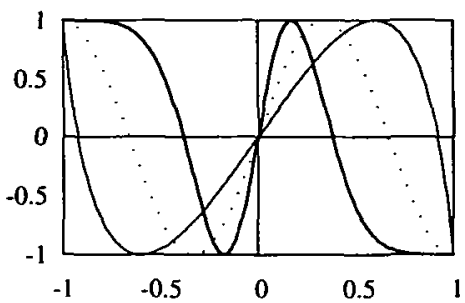

(c) $k=3$

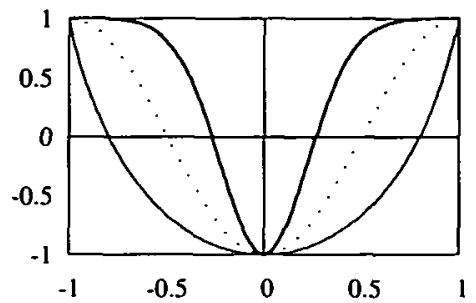

(b) $k=2$

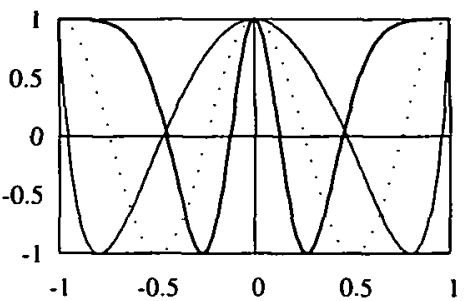

(d) $k=4$

FIGURE 1. Graphs of $\psi_{k}^{|r|}(x), r=1 / 2,1,2$, for each $k=1,2,3,4$.

For instance, Figure 1 compares the graphs of $\psi_{k}^{[r]}(x), r=1 / 2,1,2$, for each $k=1,2,3,4$. Therein the simple sigmoidal transformation $\gamma_{r}$ in $(2.1)$ was used. It is found that the higher the order $r$ is, the further concentrated on the centre $x=0$ the oscillating part of $\psi_{k}^{[r]}(x)$ is. In other words, compared with the graph of $\psi_{k}(x)=\psi_{k}^{[1]}(x)$, the oscillating part of $\psi_{k}^{[r]}(x)$ is pushed toward both endpoints $x= \pm 1$ as $r$ becomes lower. Table 1 includes standard deviations,

$$
\sigma_{k}^{[r]}=\left(\frac{1}{k} \sum_{j=1}^{k} x_{k, j}^{[r]^{2}}\right)^{1 / 2}
$$

of the zeros of $\psi_{k}^{[r]}(x)$, which shows the dispersion of the zeros $x_{k, j}^{[r]}$ in (2.5) with respect to various values of $r$. Note that for all $k \geq 2$ the standard deviation of the zeros of the Chebyshev polynomial $T_{k}(x)=\cos \left[k \cos ^{-1} x\right]$ is $1 / \sqrt{2} \approx 0.707107$ which is between those of $\psi_{k}^{[1]}$ and $\psi_{k}^{[1 / 2]}$.

LEMMA 2.2. For any $r>0$ the weight function $w^{[r]}(x)$ defined in $(2.3)$ has the following properties:

(i) $w^{[r]}(-x)=w^{[r]}(x)$ with $w^{[r]}(0)=\gamma_{r}^{\prime}(1 / 2)$ and $d w^{[r]}(0) / d x=0$;

(ii) near both endpoints $x= \pm 1$ the local behaviour of $w^{[r]}(x)$ is

$$
w^{[r \mid}(x)=O\left(\left(1-x^{2}\right)^{r-1}\right) .
$$


TABLE 1. Values of the standard deviations $\sigma_{k}^{[r]}$ of the zeros of $\psi_{k}^{[r]}(x)$ with respect to the order $r$ $(k=2,10,15,20,25)$.

\begin{tabular}{cccccc}
\hline$r$ & $\sigma_{2}^{[r]}$ & $\sigma_{10}^{[r]}$ & $\sigma_{15}^{[r]}$ & $\sigma_{20}^{[r]}$ & $\sigma_{25}^{[r]}$ \\
\hline 8 & 0.068556 & 0.101124 & 0.104207 & 0.105828 & 0.106836 \\
4 & 0.136470 & 0.197435 & 0.202671 & 0.205325 & 0.206929 \\
2 & 0.267949 & 0.362632 & 0.368333 & 0.370899 & 0.372317 \\
1 & 0.500000 & 0.574456 & 0.576066 & 0.576628 & 0.576888 \\
$1 / 2$ & 0.800000 & 0.755518 & 0.755512 & 0.755511 & 0.755511 \\
$1 / 4$ & 0.975610 & 0.872584 & 0.872563 & 0.872563 & 0.872563 \\
$1 / 8$ & 0.999695 & 0.939622 & 0.936019 & 0.936257 & 0.936243 \\
\hline
\end{tabular}

Proof. Since $\gamma_{r}^{\prime}(y)=\gamma_{r}^{\prime}(1-y)$ it follows that

$$
w^{[r]}(-x)=\gamma_{r}^{\prime}\left(\frac{1-x}{2}\right)=\gamma_{r}^{\prime}\left(\frac{1+x}{2}\right)=w^{[r]}(x) .
$$

Differentiating (S2) twice gives $\gamma_{r}^{\prime \prime}(y)+\gamma_{r}^{\prime \prime}(1-y)=0$ so that

$$
\frac{d w^{[r]}}{d x}(x)+\frac{d w^{[r]}}{d x}(-x)=\frac{1}{2}\left\{\gamma_{r}^{\prime \prime}\left(\frac{1+x}{2}\right)+\gamma_{r}^{\prime \prime}\left(\frac{1-x}{2}\right)\right\}=0 .
$$

Thus we have $d w^{[r]}(0) / d x=0$ by substituting $x=0$.

For property (ii), referring to (S5) in Definition 2.1 , we can see that

$$
w^{[r]}(x)= \begin{cases}O\left((1+x)^{r-1}\right) & \text { for } x \text { near }-1 \\ \gamma_{r}^{\prime}((1+x) / 2)=\gamma_{r}^{\prime}((1-x) / 2)=O\left((1-x)^{r-1}\right) & \text { for } x \text { near } 1 .\end{cases}
$$

This completes the proof.

\section{Sigmoidal cosine series (SCS)}

We recall that a function $f$ is called piecewise continuous if it has at most a finite number of points of discontinuity and, in addition, the one-sided limits exist at each point of discontinuity. Furthermore we introduce the definition of the piecewise smooth function as follows.

DEFINITION 3.1. We say that a function $f$ is piecewise smooth on $[a, b]$ if and only if

(1) $f$ is piecewise continuous on $[a, b]$; 
(2) the first derivative $f^{\prime}$ is piecewise continuous on $[a, b]$. More precisely, the one-sided derivatives of $f$ at each point in $[a, b]$ exist in a sense as follows:

(i) At each $x \in(a, b]$ the following limit exists:

$$
\lim _{h \rightarrow 0_{+}} \frac{f\left(x_{-}\right)-f(x-h)}{h} .
$$

(ii) At each $x \in[a, b)$ the following limit exists:

$$
\lim _{h \rightarrow 0_{+}} \frac{f(x+h)-f\left(x_{+}\right)}{h} .
$$

For a piecewise continuous function $f$ on an interval $[-1,1]$ we consider a series, called a sigmoidal cosine series (SCS),

$$
S^{[r]}(f ; x)=\sum_{n=0}^{\infty} c_{n} \psi_{n}^{[r]}(x)=\frac{c_{0}}{\sqrt{2}}+\sum_{n=1}^{\infty} c_{n} \cos \left[\pi n \gamma_{r}\left(\frac{1+x}{2}\right)\right],
$$

where $-1 \leq x \leq 1$ and the coefficient $c_{n}$ is

$$
c_{n}=\int_{-1}^{1} w^{[r]}(t) \psi_{n}^{[r]}(t) f(t) d t
$$

We denote by $S_{N}^{[r]}(f ; x)=\sum_{n=0}^{N} c_{n} \psi_{n}^{[r]}(x)$ the partial sum of the SCS, $S^{[r]}(f ; x)$.

From now on we will show a pointwise convergence of the present series given in (3.1). If we set $\xi=\gamma_{r}((1+x) / 2), 0 \leq \xi \leq 1$, then the coefficients in (3.2) become

$$
c_{n}=2 \int_{0}^{1} \cos [\pi n s] f\left(2 \gamma_{r}^{-1}(s)-1\right) d s, \quad n \geq 1,
$$

with $c_{0}=\sqrt{2} \int_{0}^{1} f\left(2 \gamma_{r}^{-1}(s)-1\right) d s$. Thus from (3.1) we have

$$
\begin{aligned}
S_{N}^{[r]}(f ; x) & =2 \int_{0}^{1} f\left(2 \gamma_{r}^{-1}(s)-1\right) \sum_{n=0}^{N} \cos [\pi n s] \cos [\pi n \xi] d s \\
& =\int_{0}^{1} f\left(2 \gamma_{r}^{-1}(s)-1\right) \sum_{n=0}^{N}\{\cos [\pi n(s-\xi)]+\cos [\pi n(s+\xi)]\} d s \\
& =\int_{0}^{1} f\left(2 \gamma_{r}^{-1}(s)-1\right) \tilde{D}_{N}(s ; \xi) d s
\end{aligned}
$$

where $\sum_{n=0}^{\prime N} a_{n}=a_{0} / 2+\sum_{n=0}^{N} a_{n}$ and $\tilde{D}_{N}(s ; \xi)$ is a kernel defined by

$$
\tilde{D}_{N}(s ; \xi)=\sum_{n=0}^{N}\{\cos [\pi n(s-\xi)]+\cos [\pi n(s+\xi)]\} .
$$


Using an identity

$$
\sum_{n=0}^{N} \cos [\pi n \theta]=\frac{\sin \pi(N+1 / 2) \theta}{2 \sin \frac{\pi}{2} \theta},
$$

we rewrite $\tilde{D}_{N}(s ; \xi)$ as

$$
\begin{aligned}
\tilde{D}_{N}(s ; \xi)= & \frac{\sin \pi(N+1 / 2)(s-\xi)}{2 \sin \frac{\pi}{2}(s-\xi)}+\frac{\sin \pi(N+1 / 2)(s+\xi)}{2 \sin \frac{\pi}{2}(s+\xi)} \\
= & \frac{1}{2 \sin \frac{\pi}{2}(s-\xi)}\left\{\sin \frac{\pi}{2}(2 N+1)(s-\xi)\right. \\
& \left.+\sin \frac{\pi}{2}(s-\xi) \frac{\sin \pi(N+1 / 2)(s+\xi)}{\sin \frac{\pi}{2}(s+\xi)}\right\} \\
:= & \frac{1}{2 \sin \frac{\pi}{2}(s-\xi)} G_{N}(s ; \xi) .
\end{aligned}
$$

Following the procedure given in the literature [5] for the convergence analysis of the standard Fourier series, we can also obtain the convergence of the present SCS $S_{N}^{[r]}(f ; x)$ with respect to the range of $r$. First, we need some properties from the following lemma.

LEMMA 3.1. For each $0<\xi<-1$ we have:

(i) For any piecewise continuous functions $g(s)$ and $h(s)$ on the intervals $[0, \xi]$ and $[\xi, 1]$, respectively,

$$
\lim _{N \rightarrow \infty} \int_{0}^{\xi} g(s) G_{N}(s ; \xi) d s=\lim _{N \rightarrow \infty} \int_{\xi}^{1} h(s) G_{N}(s ; \xi) d s=0 .
$$

(ii) For any integer $N \geq 1$,

$$
\int_{0}^{\xi} \tilde{D}_{N}(s ; \xi) d s=\xi+\sum_{n=1}^{N} \frac{1}{\pi n} \sin (2 \pi n \xi)
$$

and

$$
\int_{\xi}^{1} \tilde{D}_{N}(s ; \xi) d s=1-\xi-\sum_{n=1}^{N} \frac{1}{\pi n} \sin (2 \pi n \xi) .
$$

In addition, and consequently, $\int_{0}^{1} \tilde{D}_{N}(s ; \xi) d s=1$.

(iii)

$$
\sum_{n=1}^{\infty}(\pi n)^{-1} \sin (2 \pi n \xi)=1 / 2-\xi
$$


ProOF. From (3.6),

$$
\begin{aligned}
\int_{0}^{\xi} g(s) & G_{N}(s ; \xi) d s \\
= & \int_{0}^{\xi} g(s)\left\{\sin \frac{\pi}{2}(2 N+1)(s-\xi)\right. \\
& \left.+\sin \frac{\pi}{2}(s-\xi) \frac{\sin \frac{\pi}{2}(2 N+1)(s+\xi)}{\sin \frac{\pi}{2}(s+\xi)}\right\} d s \\
= & \int_{0}^{\xi} g(s)\left\{1+\frac{\sin \frac{\pi}{2}(s-\xi)}{\sin \frac{\pi}{2}(s+\xi)}\right\} \cos (N+1 / 2) \xi \sin \pi(N+1 / 2) s d s \\
& -\int_{0}^{\xi} g(s)\left\{1-\frac{\sin \frac{\pi}{2}(s-\xi)}{\sin \frac{\pi}{2}(s+\xi)}\right\} \sin (N+1 / 2) \xi \cos \pi(N+1 / 2) s d s .
\end{aligned}
$$

If we define two functions

$$
k_{1}(s):= \begin{cases}g(s)\left\{1+\frac{\sin \frac{\pi}{2}(s-\xi)}{\sin \frac{\pi}{2}(s+\xi)}\right\} \cos (N+1 / 2) \xi, & 0 \leq s \leq \xi, \\ 0, & \xi<s \leq 1,\end{cases}
$$

and

$$
k_{2}(s):= \begin{cases}g(s)\left\{1-\frac{\sin \frac{\pi}{2}(s-\xi)}{\sin \frac{\pi}{2}(s+\xi)}\right\} \sin (N+1 / 2) \xi, & 0 \leq s \leq \xi, \\ 0, & \xi<s \leq 1,\end{cases}
$$

then

$$
\int_{0}^{\xi} g(s) G_{N}(s ; \xi) d s=\int_{0}^{1} k_{1}(s) \sin \frac{\pi(2 N+1) s}{2} d s-\int_{0}^{1} k_{2}(s) \cos \frac{\pi(2 N+1) s}{2} d s .
$$

Since $k_{1}(s)$ and $k_{2}(s)$ are piecewise continuous on $[0,1]$, the Riemann-Lebesgue lemma implies $\lim _{N \rightarrow \infty} \int_{0}^{\xi} g(s) G_{N}(s ; \xi) d s=0$.

Similarly it also follows that $\lim _{N \rightarrow \infty} \int_{\xi}^{1} h(s) G_{N}(s ; \xi) d s=0$.

For property (ii), we have from (3.5)

$$
\begin{aligned}
\int_{0}^{\xi} \tilde{D}_{N}(s ; \xi) d s & =2 \int_{0}^{\xi} \sum_{n=0}^{N} \cos [\pi n s] \cos [\pi n \xi] d s \\
& =2\left\{\frac{\xi}{2}+\sum_{n=1}^{N} \cos [\pi n \xi] \int_{0}^{\xi} \cos [\pi n s] d s\right\} \\
& =\xi+\sum_{n=1}^{N} \frac{1}{\pi n} \sin [2 \pi n \xi] .
\end{aligned}
$$


Similarly it follows that

$$
\begin{aligned}
\int_{\xi}^{1} \tilde{D}_{N}(s ; \xi) d s & =2\left\{\frac{1-\xi}{2}+\sum_{n=1}^{N} \cos [\pi n \xi] \int_{\xi}^{1} \cos [\pi n s] d s\right\} \\
& =1-\xi-\sum_{n=1}^{N} \frac{1}{\pi n} \sin [2 \pi n \xi] .
\end{aligned}
$$

Finally, for property (iii) we define a function

$$
A(\xi):=\xi+\sum_{n=1}^{\infty} \frac{1}{\pi n} \sin (2 \pi n \xi) .
$$

Then from the formula (FI II 559) in [6], we have

$$
\sum_{n=1}^{\infty} \frac{1}{n} \sin (n x)=\tan ^{-1}\left(\frac{\sin x}{1-\cos x}\right), \quad 0<x<2 \pi,
$$

and $A(\xi)$ becomes

$$
A(\xi)=\xi+\frac{1}{\pi} \tan ^{-1}(a(\xi)), \quad a(\xi)=\frac{\sin 2 \pi \xi}{1-\cos 2 \pi \xi} .
$$

Since

$$
a^{\prime}(\xi)=\frac{2 \pi(\cos 2 \pi \xi-1)}{(1-\cos 2 \pi \xi)^{2}}
$$

and

$$
1+a(\xi)^{2}=\frac{(1-\cos 2 \pi \xi)^{2}+\sin ^{2} 2 \pi \xi}{(1-\cos 2 \pi \xi)^{2}}=\frac{-2(\cos 2 \pi \xi-1)}{(1-\cos 2 \pi \xi)^{2}}
$$

it follows that

$$
A^{\prime}(\xi)=1+\frac{1}{\pi} \frac{a^{\prime}(\xi)}{1+a(\xi)^{2}} \equiv 0 .
$$

Therefore $A(\xi) \equiv$ constant. But $A(1 / 2)=1 / 2$, and thus we have $A(\xi) \equiv 1 / 2$ for all $0<\xi<1$.

Owing to the results above we can directly induce the pointwise convergence of $S_{N}^{|r|}(f ; x)$ as shown in Theorem 3.2, which is parallel to Dirichlet's theorem [5,9] for the partial sum of the Fourier series.

THEOREM 3.2. Let $f$ be a piecewise smooth function on the interval $[-1,1]$. Then for any $r>0$ and for each $-1<x<1$, the partial sum of the SCS of $f$ converges as

$$
\lim _{N \rightarrow \infty} S_{N}^{|r|}(f ; x)=\frac{1}{2}\left\{f\left(x_{-}\right)+f\left(x_{+}\right)\right\} .
$$


In addition, at the endpoints $x= \pm 1$, for $0<r \leq 1$,

$$
\lim _{N \rightarrow \infty} S_{N}^{[r]}(f ; 1)=f\left(1_{-}\right), \quad \lim _{N \rightarrow \infty} S_{N}^{[r]}(f ;-1)=f\left(-1_{+}\right),
$$

respectively.

PROOF. For a fixed $-1<x<1$ we define a function $g_{1}(s)$ with $\xi=\gamma_{r}((1+x) / 2)$, $0<\xi<1$, as

$$
g_{1}(s):=\frac{f\left(2 \gamma_{r}^{-1}(s)-1\right)-f\left(x_{-}\right)}{2 \sin \frac{\pi}{2}(s-\xi)} .
$$

Then

$$
\lim _{s \rightarrow \xi_{-}} g_{1}(s)=\lim _{s \rightarrow \xi_{-}} \frac{f\left(x_{-}\right)-f\left(2 \gamma_{r}^{-1}(s)-1\right)}{\xi-s} \cdot \frac{\xi-s}{2 \sin \frac{\pi}{2}(\xi-s)} .
$$

Noting that for any $r>0$ the function $\gamma_{r}^{-1}$ is smooth and strictly increasing on $(0,1)$, from condition (i) in Definition 3.1 (2) we can see that $g_{1}(s)$ is piecewise continuous on the interval $[0, \xi]$. Therefore, from Lemma 3.1 (i),

$$
\lim _{N \rightarrow \infty} \int_{0}^{\xi} g_{1}(s) G_{N}(s ; \xi) d s=0 .
$$

Since $G_{N}(s ; \xi)=2 \sin \frac{\pi}{2}(s-\xi) \tilde{D}_{N}(s ; \xi)$ from (3.6), it follows that

or

$$
\lim _{N \rightarrow \infty}\left[\int_{0}^{\xi} f\left(2 \gamma_{r}^{-1}(s)-1\right) \tilde{D}_{N}(s ; \xi) d s-f\left(x_{-}\right) \int_{0}^{\xi} \tilde{D}_{N}(s ; \xi) d s\right]=0,
$$

$$
\lim _{N \rightarrow \infty} \int_{0}^{\xi} f\left(2 \gamma_{r}^{-1}(s)-1\right) \tilde{D}_{N}(s ; \xi) d s=\left\{\lim _{N \rightarrow \infty} \int_{0}^{\xi} \tilde{D}_{N}(s ; \xi) d s\right\} f\left(x_{-}\right) .
$$

Similarly, using a function $g_{2}(s)$ defined as

$$
g_{2}(s):=\frac{f\left(2 \gamma_{r}^{-1}(s)-1\right)-f\left(x_{+}\right)}{2 \sin \frac{\pi}{2}(s-\xi)},
$$

we can see that Lemma 3.1 (i) and condition (ii) in Definition 3.1 (2) imply

$$
\lim _{N \rightarrow \infty} \int_{\xi}^{1} f\left(2 \gamma_{r}^{-1}(s)-1\right) \tilde{D}_{N}(s ; \xi) d s=\left\{\lim _{N \rightarrow \infty} \int_{\xi}^{1} \tilde{D}_{N}(s ; \xi) d s\right\} f\left(x_{+}\right) .
$$

Therefore, if we set $\alpha:=\lim _{N \rightarrow \infty} \int_{0}^{\xi} \tilde{D}_{N}(s ; \xi) d s$ and $\beta:=\lim _{N \rightarrow \infty} \int_{\xi}^{1} \tilde{D}_{N}(s ; \xi) d s$, then from (3.4) we have

$$
\begin{aligned}
\lim _{N \rightarrow \infty} & S_{N}^{[r]}(f ; x) \\
& =\lim _{N \rightarrow \infty}\left[\int_{0}^{\xi} f\left(2 \gamma_{r}^{-1}(s)-1\right) \tilde{D}_{N}(s ; \xi) d s+\int_{\xi}^{1} f\left(2 \gamma_{r}^{-1}(s)-1\right) \tilde{D}_{N}(s ; \xi) d s\right] \\
& =\alpha f\left(x_{-}\right)+\beta f\left(x_{+}\right) .
\end{aligned}
$$


However, from Lemma 3.1 (ii)-(iii), for all $-1<x<1$ (that is, $0<\xi<1$ ),

$$
\alpha=\xi+\sum_{n=1}^{\infty} \frac{1}{\pi n} \sin (2 \pi n \xi)=\frac{1}{2} .
$$

On the other hand, since $\int_{0}^{1} \tilde{D}_{N}(s ; \xi) d s=1$ it is clear that

$$
\alpha+\beta=\lim _{N \rightarrow \infty} \int_{0}^{1} \tilde{D}_{N}(s ; \xi) d s=1,
$$

that is, $\beta=1 / 2$. This results in Equation (3.7).

For the case of the endpoint $x=1$ (that is, $\xi=1$ ) the condition $0<r \leq 1$ implies that, in the formula (3.9), $\lim _{s \rightarrow \xi_{-}} g_{1}(s)$ exists because $\left(\gamma_{r}^{-1}\right)^{\prime}(1)$ exists and thus $f\left(1_{-}\right)-f\left(2 \gamma_{r}^{-1}(s)-1\right)=O(1-s)$ for $s$ near 1 . Since $g_{1}$ is now piecewise continuous on $[0,1]$ it follows that

$$
\lim _{N \rightarrow \infty} S_{N}^{[r]}(f ; 1)=\left\{\lim _{N \rightarrow \infty} \int_{0}^{1} \tilde{D}_{N}(s ; 1) d s\right\} f\left(1_{-}\right)=f\left(1_{-}\right) .
$$

When $x=-1$ (that is, $\xi=0$ ) we have similarly that

$$
\lim _{N \rightarrow \infty} S_{N}^{[r]}(f ;-1)=\left\{\lim _{N \rightarrow \infty} \int_{0}^{1} \tilde{D}_{N}(s ; 0) d s\right\} f\left(-1_{+}\right)=f\left(-1_{+}\right) .
$$

THEOREM 3.3. Let $f$ be continuous and piecewise smooth on $[-1,1]$. Then for any $0<r \leq 1$ the SCS of $f, S^{[r]}(f ; x)$, converges uniformly to $f(x)$ on $[-1,1]$.

Proof. Define a function

$$
h(s):=f\left(2 \gamma_{r}^{-1}(s)-1\right), \quad 0 \leq s \leq 1 .
$$

Since $\left(\gamma_{r}^{-1}\right)^{\prime}(s)=O\left(s^{1 / r-1}\right)$ near $s=0$ and $\left(\gamma_{r}^{-1}\right)^{\prime}(s)=O\left((1-s)^{1 / r-1}\right)$ near $s=1$, the derivative $h^{\prime}(s)=2\left(\gamma_{r}^{-1}\right)^{\prime}(s) f^{\prime}\left(2 \gamma_{r}^{-1}(s)-1\right)$ is piecewise continuous on $[0,1]$ for any $0<r \leq 1$, and thus $h(s)$ is continuous and piecewise smooth on $[0,1]$.

From the formula (3.3) the coefficient of the SCS of $f$ can be rewritten by

$$
c_{n}=2 \int_{0}^{1} \cos [n \pi s] h(s) d s
$$

which is the Fourier cosine coefficient of $h$ on the interval $[0,1]$. Therefore the series $\sum_{n=1}^{\infty}\left|c_{n}\right|$ is convergent and thus, from (3.1) and the Weierstrass $M$-test, the series

$$
\lim _{N \rightarrow \infty} S_{N}^{(r)}(f ; x)=\frac{c_{0}}{\sqrt{2}}+\sum_{n=1}^{\infty} c_{n} \cos \left[\pi n \gamma_{r}\left(\frac{1+x}{2}\right)\right]
$$


converges uniformly on $[-1,1]$. Furthermore, from Theorem 3.2 , we can see that $S^{[r]}(f ; x)$ converges uniformly to $\left\{f\left(x_{-}\right)+f\left(x_{+}\right)\right\} / 2=f(x)$ on $(-1,1)$ and to $f( \pm 1)$ at the endpoints $x= \pm 1$.

The following theorem shows a connection between the differentiability property of a function and the rate of convergence of its SCS according to the order $r$.

THEOREM 3.4. Let $f \in C^{k-1}([-1,1]), k \geq 1$, and let $f^{(k)}$ be piecewise continuous on $[-1,1]$. Then for any $0<r \leq 1 / k$ the SCS coefficients $c_{n}$ of $f$ satisfy $n^{k} c_{n} \rightarrow 0$ as $n \rightarrow \infty$.

Proof. The $k$ th derivative $h^{(k)}(s)$ of the function $h(s)$ defined in (3.11) contains $\left(\gamma_{r}^{-1}\right)^{(j)}$ and $f^{(j)}$ for $j=1,2, \ldots k$. Since near $s=0$ and $s=1$, respectively,

$$
\left(\gamma_{r}^{-1}\right)^{(j)}(s)=O\left(s^{1 / r-j}\right), \quad\left(\gamma_{r}^{-1}\right)^{(j)}(s)=O\left((1-s)^{1 / r-j}\right),
$$

the condition $0<r \leq 1 / k$ implies that the function $h^{(k)}(s)$ is piecewise continuous on $[0,1]$.

Further, we note that for any $0<r \leq 1 / k$,

$$
h^{(j)}(0)=h^{(j)}(1)=0, \quad j=1,2, \ldots, k-1 .
$$

Thus, by using integration by parts $k$ times, we can see that the coefficients of the SCS given in (3.3) become for all $n \geq 1$

$$
c_{n}= \begin{cases}\frac{(-1)^{(1+k) / 2}}{(n \pi)^{k}} 2 \int_{0}^{1} \sin [n \pi s] h^{(k)}(s) d s, & k \text { is odd, } \\ \frac{(-1)^{k / 2}}{(n \pi)^{k}} 2 \int_{0}^{1} \cos [n \pi s] h^{(k)}(s) d s, & k \text { is even. }\end{cases}
$$

Since the integrals in these formulas are the Fourier coefficients of $h^{(k)}$ which is piecewise continuous on $[0,1]$, they vanish as $n \rightarrow \infty$ by the Riemann-Lebesgue lemma. Therefore it follows that $n^{k} c_{n} \rightarrow 0$ as $n \rightarrow \infty$.

The next theorem shows the convergence of the SCS for a function which is not piecewise smooth on the interval $[-1,1]$, in general, but whose derivative may have a weak singularity at the endpoints.

THEOREM 3.5. Let $f$ be a piecewise smooth function on any interval $[a, b]$ for all $a$, $b$ such that $-1<a<b<1$ and assume that near $x=1$ and $x=-1$ the behaviour of $f$ is, respectively,

$$
f(x) \sim A_{1}+B_{1}(1-x)^{\eta_{1}}, \quad f(x) \sim A_{2}+B_{2}(1+x)^{\eta_{2}},
$$

where $\eta_{1}, \eta_{2}>0$, and $A_{i}$ and $B_{i}$ are constants such that $B_{1}^{2}+B_{2}^{2} \neq 0$. Then for any $0<r \leq \eta=\min \left\{\eta_{1}, \eta_{2}\right\}$ we have: 
(i) The partial sum $S_{N}^{[r]}(f ; x)$ converges on the interval $[-1,1]$ in the form of (3.7) and (3.8).

(ii) If, in addition, $f$ is continuous on $[-1,1]$ then the series $S^{[r]}(f ; x)$ converges uniformly to $f(x)$ on $[-1,1]$.

In particular if $B_{1}=B_{2}=0$, the results above hold for all $r>0$.

PROOF. Referring to the proof of Theorem 3.2, we only have to consider the cases of $x= \pm 1$. First, let $x=1$ or $\xi=1$ therein, then from (3.9)

$$
\lim _{s \rightarrow 1_{-}} g_{1}(s)=\lim _{s \rightarrow 1_{-}} \frac{f\left(1_{-}\right)-f\left(2 \gamma_{r}^{-1}(s)-1\right)}{1-s} \cdot \frac{1-s}{2 \sin \frac{\pi}{2}(1-s)} .
$$

Since near $s=1$, from (3.12), $f\left(1_{-}\right)-f\left(2 \gamma_{r}^{-1}(s)-1\right)=O\left((1-s)^{\eta_{1} / r}\right)$, we have

$$
\lim _{s \rightarrow 1_{-}} \frac{f\left(1_{-}\right)-f\left(2 \gamma_{r}^{-1}(s)-1\right)}{1-s}=k(1-s)^{\eta_{1} / r-1},
$$

where $k$ is a constant. Thus, for any $0<r \leq \eta_{1}, g_{1}(s)$ is piecewise continuous on $[0,1]$ so that

$$
\lim _{N \rightarrow \infty} S_{N}^{[r]}(f ; 1)=\lim _{N \rightarrow \infty} \int_{0}^{1} f\left(2 \gamma_{r}^{-1}(s)-1\right) \tilde{D}_{N}(s ; 1) d s=f\left(1_{-}\right)=A_{1} .
$$

When $x=-1$ or $\xi=0$, similarly it can be seen that the function $g_{2}(s)$ defined in (3.10) is piecewise continuous on $[0,1]$ for $0<r \leq \eta_{2}$. Therefore

$$
\lim _{N \rightarrow \infty} S_{N}^{[r]}(f ;-1)=\lim _{N \rightarrow \infty} \int_{0}^{1} f\left(2 \gamma_{r}^{-1}(s)-1\right) \tilde{D}_{N}(s ; 0) d s=f\left(-1_{+}\right)=A_{2},
$$

which completes the proof of property (i).

On the other hand, for the function $h(s)$ defined in (3.11), its derivative

$$
h^{\prime}(s)=2\left(\gamma_{r}^{-1}\right)^{\prime}(s) f^{\prime}\left(2 \gamma_{r}^{-1}(s)-1\right)
$$

behaves like

$$
h^{\prime}(s)= \begin{cases}O\left((1-s)^{\eta_{1} / r-1}\right), & \text { near } s=1, \\ O\left(s^{\eta_{2} / r-1}\right), & \text { near } s=0 .\end{cases}
$$

Then for any $r \leq \eta=\min \left\{\eta_{1}, \eta_{2}\right\}$ the function $h(s)$ is piecewise smooth on $[0,1]$, and thus Theorem 3.3 implies property (ii).

In a particular case of $B_{1}=B_{2}=0$ the local behaviour of the functions $g_{1}, g_{2}$ and $h$ near both endpoints $x= \pm 1(\xi=1,0)$ does not matter in the statements above. Thus properties (i) and (ii) are true independently of $r>0$. 


\section{The Gibbs phenomenon}

If a function $f$ has a discontinuity at $x_{0}$ then any series expansion such as the Fourier series, $S^{\mathrm{F}}(f ; x)$, or the present $\operatorname{SCS}, S^{[r]}(f ; x)$, cannot converge uniformly on any interval containing $x_{0}$ because the series is composed of continuous functions. The lack of uniformity of a series expansion reveals itself in a particular way known as the Gibbs phenomenon [5, 7,9]. That is, as one adds on more and more terms, the partial sums of the series expansion overshoot and undershoot $f$ near the discontinuity $x_{0}$ and thus develop spikes that tend to zero in width but not in height.

Taking account of the behaviour of $\psi_{k}^{[r]}$ in Figure 1, we expect that the SCS $S^{[r]}$ based on $\psi_{k}^{[r]}$ may further reduce the spikes in width by controlling the value of $r$. For example, we consider a simple piecewise continuous function,

$$
g(x)=\left\{\begin{array}{lr}
0, & -1 \leq x<0 \\
1, & 0<x \leq 1,
\end{array}\right.
$$

with a discontinuity $x_{0}=0$. Since this is the case of $B_{1}=B_{2}=0$ in Theorem 3.5 any $r>0$ is available for the pointwise convergence of the $\operatorname{SCS} S^{[r]}(g ; x)$. The partial sum of $S^{[r]}(g ; x)$ takes the form

$$
S_{N}^{[r]}(g ; x)=\frac{c_{0}}{\sqrt{2}}+\sum_{k=1}^{N}\left\{c_{2 k-1} \psi_{2 k-1}^{[r]}(x)+c_{2 k} \psi_{2 k}^{[r]}(x)\right\},
$$

in which the coefficients are, for each $k \geq 1$,

$$
c_{2 k-1}=\int_{0}^{1} w^{[r]}(x) \psi_{2 k-1}^{[r]}(x) d x=2 \int_{1 / 2}^{1} \cos [(2 k-1) \pi s] d s=2 \frac{(-1)^{k}}{(2 k-1) \pi}
$$

and

$$
c_{2 k}=\int_{0}^{1} w^{[r]}(x) \psi_{2 k}^{[r]}(x) d x=2 \int_{1 / 2}^{1} \cos [(2 k) \pi s] d s=0
$$

with

$$
c_{0}=\frac{1}{\sqrt{2}} \int_{0}^{1} w^{[r]}(x) d x=\frac{1}{\sqrt{2}} .
$$

Therefore (4.2) becomes

$$
S_{N}^{[r]}(g ; x)=\frac{1}{2}+\frac{2}{\pi} \sum_{k=1}^{N} \frac{(-1)^{k}}{(2 k-1)} \cos \left[(2 k-1) \pi \gamma_{r}\left(\frac{1+x}{2}\right)\right] .
$$

Referring to the literature [7], we investigate the Gibbs phenomenon of the present $\mathrm{SCS}$ for the function $g$ given in (4.1). If we set

$$
\gamma_{r}\left(\frac{1+x}{2}\right)=\frac{1+t}{2}, \quad-1 \leq t \leq 1,
$$


then $S_{N}^{[r]}$ in (4.3) becomes

$$
\begin{aligned}
S_{N}^{[r]}(g ; x) & =\frac{1}{2}+\sum_{k=1}^{N} \frac{\sin [(2 k-1) \pi t / 2]}{(2 k-1) \pi / 2} \\
& =\frac{1}{2}+\sum_{k=1}^{N} \int_{0}^{t} \cos \left[\frac{2 k-1}{2} \pi \tau\right] d \tau=\frac{1}{2}+\frac{1}{2} \int_{0}^{t} \frac{\sin (N \pi \tau)}{\sin (\pi \tau / 2)} d \tau .
\end{aligned}
$$

The last equality was due to the equation

$$
\sum_{k=1}^{N} \cos \left[\frac{2 k-1}{2} \pi \tau\right]=\frac{\sin (N \pi \tau)}{2 \sin (\pi \tau / 2)} .
$$

By the change of variables $N \pi \tau=y$, we have

$$
S_{N}^{[r]}(g ; x)=\frac{1}{2}+\frac{1}{2} \int_{0}^{N \pi t} \frac{\sin y}{\sin (y / 2 N)} \frac{1}{N \pi} d y .
$$

If we differentiate $S_{N}^{[r]}(g ; x)$ with respect to $x$, then

$$
\frac{d S_{N}^{[r]}(g ; x)}{d x}=\frac{1}{2} w^{[r]}(x) \frac{\sin (N \pi t)}{\sin (t \pi / 2)} .
$$

Thus $S_{N}^{[r]}(g ; x)$ has extreme values at the critical points, say, $x_{k}=2 \gamma_{r}^{-1}\left(\left(1+t_{k}\right) / 2\right)-1$ with $t_{k}=k / N$ for integers $k= \pm 1, \pm 2, \ldots, \pm N-1$. That is,

$$
x_{k}=2 \gamma_{r}^{-1}\left(\frac{1}{2}+\frac{k}{2 N}\right)-1 \text {. }
$$

This implies that as $r>1$ grows larger, the critical points $x_{k}$ of the SCS become further clustered toward the centre $x=0$ while those of the Fourier series, $t_{k}$ are uniformly distributed.

Moreover, for $r>1$, the width $\Delta x_{k}:=x_{k+1}-x_{k}$ of the spike becomes

$$
\Delta x_{k}=2\left\{\gamma_{r}^{-1}\left(\frac{1}{2}+\frac{k+1}{2 N}\right)-\gamma_{r}^{-1}\left(\frac{1}{2}+\frac{k}{2 N}\right)\right\} \sim \frac{\left(\gamma_{r}^{-1}\right)^{\prime}(1 / 2)}{N},
$$

for $k \ll N$ due to the asymptotic behaviour,

$$
\gamma_{r}^{-1}(1 / 2+\eta) \sim 1 / 2+\left(\gamma_{r}^{-1}\right)^{\prime}(1 / 2) \eta,
$$

for small $\eta$. For instance if the simple sigmoidal transformation given in (2.1) is employed for $\gamma_{r}$ then, since $\left(\gamma_{r}^{-1}\right)^{\prime}(1 / 2)=\left(\gamma_{1 / r}\right)^{\prime}(1 / 2)=1 / r,(4.5)$ becomes

$$
\Delta x_{k} \sim 1 /(r N) \text {. }
$$


That is, even if $N$ is not very large, the widths of the spikes near the discontinuity $x_{0}=0$ are decreasing as the order $r>1$ grows.

Set $t_{N}^{*}=t_{1}=1 / N$ and $x_{N}^{*}=x_{1}=2 \gamma_{r}^{-1}(1 / 2+1 /(2 N))-1$ at which the Fourier series $S_{N}^{\mathrm{F}}(g ; x)$ and the $\operatorname{SCS} S_{N}^{[r]}(g ; x)$, respectively, have maxima or the highest spikes. It is well known that $S_{N}^{\mathrm{F}}\left(g ; t_{N}^{*}\right) \sim 1 / 2+(1 / \pi) \operatorname{Si}(\pi)=1.0895$ for sufficiently large $N$ $[7,9]$. Simultaneously, from (4.4), it also follows that

$$
S_{N}^{[r]}\left(g ; x_{N}^{*}\right) \sim \frac{1}{2}+\frac{1}{\pi} \int_{0}^{\pi} \frac{\sin y}{y} d y=\frac{1}{2}+\frac{1}{\pi} \operatorname{Si}(\pi)
$$

for sufficiently large $N$. It should be noted that no matter how large $N$ may be, the error $S_{N}^{\mathrm{F}}\left(g ; t_{N}^{*}\right)-g\left(0_{+}\right)=S_{N}^{[r]}\left(g ; x_{N}^{*}\right)-g\left(0_{+}\right)=0.0895$ is inevitable.

On the other hand, in order to search for the height of the spike of $S_{N}^{[r]}(g ; x)$ at the point $x=t_{N}^{*}$, let $N$ be large enough. Then

$$
\gamma_{r}\left(\frac{1}{2}+\frac{1}{2 N}\right) \sim \frac{1}{2}+\gamma_{r}^{\prime}\left(\frac{1}{2}\right) \frac{1}{2 N} \quad \text { and } \quad \sin \left(\frac{y}{2 N}\right) \sim \frac{y}{2 N} \text {. }
$$

Therefore we have from (4.4)

$$
\begin{aligned}
S_{N}^{[r]}\left(g ; t_{N}^{*}\right) & =\frac{1}{2}+\frac{1}{2} \int_{0}^{N \pi\left\{2 \gamma_{r}(1 / 2+1 /(2 N))-1\right\}} \frac{\sin y}{\sin (y / 2 N)} \frac{1}{N \pi} d y \\
& \sim \frac{1}{2}+\frac{1}{\pi} \int_{0}^{\gamma_{r}^{\prime}(1 / 2) \pi} \frac{\sin y}{y} d y=\frac{1}{2}+\frac{1}{\pi} \operatorname{Si}\left(\gamma_{r}^{\prime}(1 / 2) \pi\right),
\end{aligned}
$$

which approaches the value $g\left(0_{+}\right)=1$ for large $r$ because the sine integral $\operatorname{Si}(x)$ converges to $\pi / 2$ as $x \rightarrow \infty$. Particularly if $\gamma_{r}$ is substituted by the simple sigmoidal transformation given in (2.1) then (4.8) becomes $S_{N}^{[r]}\left(g ; t_{N}^{*}\right) \sim 1 / 2+(1 / \pi) \operatorname{Si}(r \pi)$.

Suppose now $N$ is not very large and let $r$ be large enough. Then $\gamma_{r}\left(\frac{1}{2}+\frac{1}{2 N}\right) \sim 1$ and we have

$$
\cos \left[(2 k-1) \pi \gamma_{r}\left(\frac{1}{2}+\frac{1}{2 N}\right)\right] \sim-1 .
$$

Thus from (4.3) it follows that

$$
S_{N}^{[r]}\left(g ; t_{N}^{*}\right) \sim \frac{1}{2}+\frac{2}{\pi} \sum_{k=1}^{N} \frac{(-1)^{k+1}}{2 k-1} .
$$

We note that

$$
\sum_{k=1}^{N} \frac{(-1)^{k+1}}{2 k-1}=\frac{\pi}{4}+\frac{(-1)^{N}}{4}\left\{\psi\left(\frac{1}{4}+\frac{N}{2}\right)-\psi\left(\frac{3}{4}+\frac{N}{2}\right)\right\},
$$




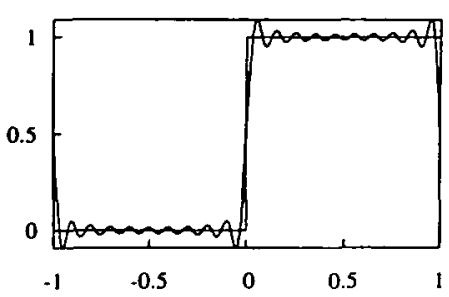

(a) Fourier series $S_{20}^{F}$

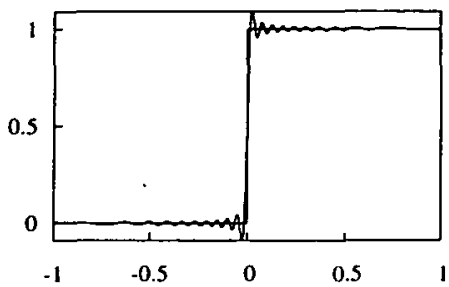

(c) $\operatorname{SCS} S_{20}^{|2|}$

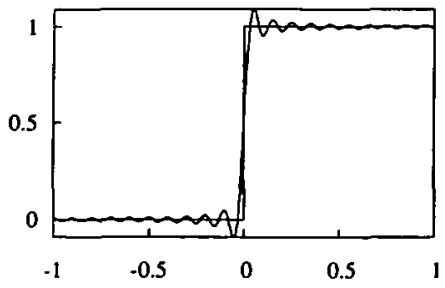

(b) $\operatorname{SCS} S_{20}^{\text {III }}$

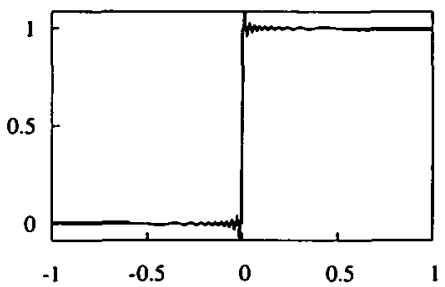

(d) $\operatorname{SCS} S_{20}^{[4]}$

FIGURE 2. The Gibbs phenomenon of the partial sums of the Fourier series $S_{N}^{\mathrm{F}}$ and the SCS $S_{N}^{(r)}$, $r=1,2,4$, for a piecewise continuous function $g(x)$ with $N=20$.

where $\psi$ is the digamma function defined by $\psi(z)=\Gamma^{\prime}(z) / \Gamma(z)$. Therefore we have

$$
S_{N}^{[r]}\left(g ; t_{N}^{*}\right) \sim 1+\frac{(-1)^{N}}{2 \pi}\left\{\psi\left(\frac{1}{4}+\frac{N}{2}\right)-\psi\left(\frac{3}{4}+\frac{N}{2}\right)\right\},
$$

for $r$ large enough.

The Gibbs phenomenon of the partial sums of the Fourier series, $S_{N}^{\mathrm{F}}$, and the SCS, $S_{N}^{[r]}$, combined with $\gamma_{r}$ given in (2.1) is illustrated in Figure 2 for $N=20$. It can be seen that as $r$ goes higher, with $N$ fixed, the widths of the spikes of $S_{N}^{[r]}$ become shorter.

\section{Periodic extension of a function on $[0,1]$}

Suppose we are interested in a function $f$ on the half-interval $[0,1]$ rather than $[-1,1]$ and we take the even extension $f_{\text {even }}$ of $f$ such that

$$
f_{\text {even }}(-x)=f(x), \quad 0 \leq x \leq 1 .
$$

Then since both $w^{[r]}$ and $\psi_{2 n}^{[r]}$ are even and $\psi_{2 n+1}^{[r]}$ is odd as shown in Theorem 2.1 (ii), the coefficients of the SCS of $f_{\text {even }}$ on $[-1,1]$ become

$$
c_{2 n}=2 \int_{0}^{1} w^{[r]}(t) f(t) \psi_{2 n}^{[r]}(t) d t, \quad c_{2 n+1}=0
$$


for all $n \geq 0$. Thus the series expansion of $f_{\text {even }}$ becomes

$$
S^{[r]}\left(f_{\text {even }} ; x\right)=\sum_{n=0}^{\infty} c_{2 n} \psi_{2 n}^{[r]}(x) .
$$

If, in addition, $\gamma_{r}$ is a 2-periodic even function on the real line $\mathbb{R}$ then so is $\psi_{2 n}^{[r]}$ since

$$
\begin{aligned}
\psi_{2 n}^{[r]}(x+2) & =\cos \left[2 n \pi \gamma_{r}(1+(1+x) / 2)\right]=\cos \left[2 n \pi \gamma_{r}(1-(1+x) / 2)\right] \\
& =\cos \left[2 n \pi\left\{1-\gamma_{r}((1+x) / 2)\right\}\right]=\psi_{2 n}^{[r]}(x)
\end{aligned}
$$

for all $x \in \mathbb{R}$. In the result, for a function $f$ satisfying the assumptions of Theorem 3.3 on the interval $[0,1]$ the series $S^{[r]}\left(f_{\text {even }} ; x\right)$ in $(5.1)$ converges uniformly to $f(x)$ on $[0,1]$, and thus it is a 2-periodic even extension of $f$ to the whole real line $\mathbb{R}$.

On the other hand, if we take the odd extension $f_{\text {odd }}$ of $f$ such that

$$
f_{\text {odd }}(-x)=-f(x), \quad 0 \leq x \leq 1,
$$

then the coefficients of the SCS of $f_{\text {odd }}$ on $[-1,1]$ become

$$
c_{2 n}=0, \quad c_{2 n+1}=2 \int_{0}^{1} w^{[r]}(t) f(t) \psi_{2 n+1}^{[r]}(t) d t
$$

for all $n \geq 0$. Thus the series expansion of $f_{\text {odd }}$ becomes

$$
S^{[r]}\left(f_{\text {odd }} ; x\right)=\sum_{n=0}^{\infty} c_{2 n+1} \psi_{2 n+1}^{[r]}(x) .
$$

However, even if $\gamma_{r}$ is 2-periodic, $\psi_{2 n+1}^{[r]}$ is not 2-periodic but 4-periodic on $\mathbb{R}$. Therefore $S^{[r]}\left(f_{\text {odd }} ; x\right)$ becomes a 4-periodic odd extension of $f$ to the real line $\mathbb{R}$.

In fact, referring to the properties (S1)-(S5) of the sigmoidal transformation $\gamma_{r}$, we can extend it to a 2-periodic even function on $\mathbb{B}$ such as

$$
\gamma_{r}^{*}(x)=\gamma_{r}(|x-2\lfloor(x+1) / 2\rfloor|), \quad x \in \mathbb{R} .
$$

It is also seen that for any even integer $m \geq 2$ a special kind of sigmoidal transformation,

$$
\gamma_{m}^{*}(x)=\frac{1}{1+\cot ^{m}(\pi x / 2)},
$$

becomes a 2-periodic even function on $\mathbb{B}$ in itself [14]. 
TABLE 2. Numerical results of $E_{N} f_{1}$ for the SCS and the Fourier series.

\begin{tabular}{ccccc}
\hline & \multicolumn{3}{c}{ SCS } & Fourier series \\
\cline { 2 - 4 }$N$ & $S_{N}^{[1 / 4]}$ & $S_{N}^{[1 / 2]}$ & $S_{N}^{[1]}$ & $S_{N}^{\mathrm{F}}$ \\
\hline 8 & $7.4 \times 10^{-1}$ & $1.5 \times 10^{-1}$ & $3.6 \times 10^{-1}$ & $3.7 \times 10^{-1}$ \\
16 & $6.5 \times 10^{-2}$ & $5.6 \times 10^{-4}$ & $2.0 \times 10^{-1}$ & $1.3 \times 10^{-1}$ \\
24 & $3.1 \times 10^{-3}$ & $7.1 \times 10^{-5}$ & $1.1 \times 10^{-1}$ & $6.5 \times 10^{-2}$ \\
32 & $1.1 \times 10^{-4}$ & $2.4 \times 10^{-5}$ & $6.9 \times 10^{-2}$ & $4.1 \times 10^{-2}$ \\
40 & $3.2 \times 10^{-6}$ & $1.0 \times 10^{-5}$ & $4.9 \times 10^{-2}$ & $2.9 \times 10^{-2}$ \\
80 & $2.9 \times 10^{-10}$ & $6.8 \times 10^{-7}$ & $1.7 \times 10^{-2}$ & $1.0 \times 10^{-2}$ \\
\hline
\end{tabular}

\section{Numerical examples}

In this section we consider several functions defined on a finite interval $[-1,1]$ to which the approximation by the partial sums of the Fourier series is not good over the whole interval. For comparison of the numerical results, we define an error such that

$$
E_{N} f:=\left[\int_{-1}^{1}\left\{f(x)-S_{N}(f ; x)\right\}^{2} d x\right]^{1 / 2},
$$

where $S_{N}(f ; x)$ denotes the $N$ th partial sum of a series expansion $S(f ; x)$ in general.

In the following examples $S_{N}^{\mathrm{F}}$ denotes a partial sum of the Fourier series $S^{\mathrm{F}}$ where

$$
S_{N}^{\mathrm{F}}(f ; x)=\frac{a_{0}}{2}+\sum_{n=1}^{N}\left\{a_{n} \cos (n \pi x)+b_{n} \sin (n \pi x)\right\}, \quad-1 \leq x \leq 1,
$$

and $a_{n}=\int_{-1}^{1} \cos (n \pi t) f(t) d t, b_{n}=\int_{-1}^{1} \sin (n \pi t) f(t) d t$.

EXAMPLE 6.1. (A smooth function having a pick near an endpoint.) Consider

$$
f_{1}(x)=e^{x} \sin \left[\frac{2 x \pi}{x^{2}-2}\right] \text {. }
$$

Since $f_{1}(x)$ takes the form of a high pick near an endpoint $x=1$, the Fourier series converges very slowly. Numerical results of the errors $E_{N} f_{1}$ for the Fourier series $S_{N}^{F}$ and the present $\operatorname{SCS} S_{N}^{[r]}, r=1 / 4,1 / 2,1$, are given in Table 2 for various $N$. It is seen that the errors of $S_{N}^{[r]}$ with $r=1 / 2$ and $1 / 4$ are much better than those of the Fourier series $S_{N}^{\mathrm{F}}$ for all $N \geq 16$. It is also seen that $S_{N}^{[1 / 41}$ exceeds $S_{N}^{[1 / 21}$ for large $N \geq 40$.

Figure 3 shows differences between $f_{1}$ and $S_{N}^{\mathrm{F}}$ in (a) and those between $f_{1}$ and $S_{N}^{[r]}$ with $r=1 / 4$ and $1 / 2$ in (b). One can observe that the approximation by $S_{N}^{[r]}$ with $r=1 / 4$ or $r=1 / 2$ dramatically improves the Fourier series approximation. 

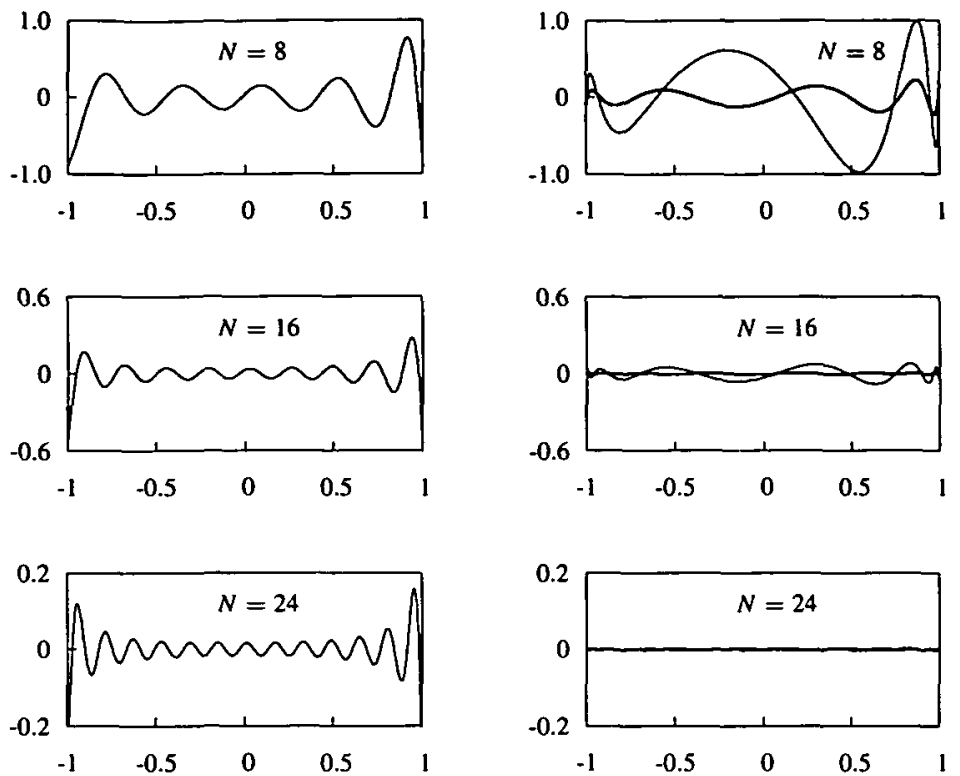

(a)

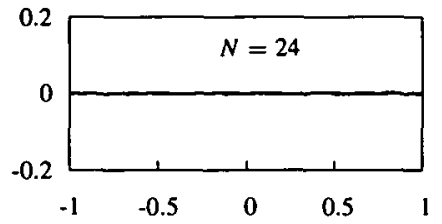

(b)

FIGURE 3. Graphs of the differences $f_{1}-S_{N}^{\mathrm{F}}$ (in (a)) and $f_{1}-S_{N}^{[r \mid}$ (in (b)). In (b) the thin line and the thick one indicate $S_{N}^{[1 / 4]}$ and $S_{N}^{[1 / 2]}$, respectively.

Superiority of the SCS in convergence seems to result from the particular feature of the base function $\psi_{n}^{[r]}$ in the vicinity of the endpoints which can be inferred from Table 1 and Figure 1 in Section 2.

EXAMPLE 6.2. (A non-piecewise smooth function.) Consider $f_{2}(x)=e^{x} \sqrt{1-x^{2}}$. The function $f_{2}(x)$ is not piecewise smooth on $[-1,1]$ because its derivative is unbounded at the endpoints $x= \pm 1$. Thus its Fourier series cannot guarantee pointwise convergence on $[-1,1]$. However Theorem 3.5 implies that the SCS $S_{N}^{[r]}$ with $0<r \leq \eta=1 / 2$ converges uniformly to $f_{2}(x)$, and thus in the following numerical fulfillment we have taken $r=1 / 4$ and $r=1 / 2$.

Table 3 includes the errors $E_{N} f_{2}$ and values of $\left(D_{N} f_{2}\right)(x)$ at the endpoints $x= \pm 1$ defined by $\left(D_{N} f\right)(x)=\left|f(x)-S_{N}(f ; x)\right|$. That is, $D_{N} f_{2}(-1)$ and $D_{N} f_{2}(1)$ denote the deviations at both endpoints which seem to be noticeable pointwise errors. Like the case of $f_{1}$, we can see that the errors $E_{N} f_{2}$ of $S_{N}^{[r]}$ with $r=1 / 2$ and $1 / 4$ are much better than those of the Fourier series $S_{N}^{\mathrm{F}}$ for all $N \geq 8$. Similarly to the results of $E_{N} f_{2}$, the deviations $D_{N} f_{2}(-1)$ and $D_{N} f_{2}(1)$ of $S_{N}^{[r]}$ highly improve those of $S_{N}^{\mathrm{F}}$.

Figure 4 shows the graphs of the differences $f_{2}-S_{N}^{F}$ in (a) and those of $f_{2}-S_{N}^{[r]}$ with $r=1 / 4$ and $1 / 2$ in (b). We can see that though the number of the series is not 
TABLE 3. Numerical results of $E_{N} f_{2},\left(D_{N} f_{2}\right)(-1)$ and $\left(D_{N} f_{2}\right)(1)$ for the SCS and the Fourier series.

\begin{tabular}{ccccc}
\hline & & \multicolumn{2}{c}{ SCS } & Fourier series \\
\cline { 2 - 4 } Errors & $N$ & $S_{N}^{[1 / 4]}$ & $S_{N}^{[1 / 2]}$ & $S_{N}^{\mathrm{F}}$ \\
\hline \multirow{5}{*}{$E_{N} f_{2}$} & 8 & $2.3 \times 10^{-2}$ & $1.4 \times 10^{-2}$ & $9.6 \times 10^{-2}$ \\
& 16 & $2.3 \times 10^{-4}$ & $4.1 \times 10^{-3}$ & $5.1 \times 10^{-2}$ \\
& 24 & $3.7 \times 10^{-6}$ & $1.9 \times 10^{-3}$ & $3.5 \times 10^{-2}$ \\
& 32 & $8.7 \times 10^{-7}$ & $1.1 \times 10^{-3}$ & $2.6 \times 10^{-2}$ \\
& 40 & $3.7 \times 10^{-7}$ & $7.5 \times 10^{-4}$ & $2.1 \times 10^{-2}$ \\
& 8 & $6.2 \times 10^{-3}$ & $9.7 \times 10^{-3}$ & $4.4 \times 10^{-1}$ \\
$\left(D_{N} f_{2}\right)(-1)$ & 24 & $1.5 \times 10^{-4}$ & $7.0 \times 10^{-3}$ & $3.3 \times 10^{-1}$ \\
& 32 & $1.2 \times 10^{-6}$ & $5.2 \times 10^{-3}$ & $2.7 \times 10^{-1}$ \\
& 40 & $6.6 \times 10^{-7}$ & $3.1 \times 10^{-3}$ & $2.4 \times 10^{-1}$ \\
& 8 & $1.9 \times 10^{-2}$ & $1.3 \times 10^{-1}$ & $2.2 \times 10^{-1}$ \\
& 16 & $1.8 \times 10^{-4}$ & $6.7 \times 10^{-2}$ & $3.3 \times 10^{-1}$ \\
$\left(D_{N} f_{2}\right)(1)$ & 24 & $2.9 \times 10^{-5}$ & $4.5 \times 10^{-2}$ & $2.7 \times 10^{-1}$ \\
& 32 & $1.3 \times 10^{-5}$ & $3.4 \times 10^{-2}$ & $2.4 \times 10^{-1}$ \\
& 40 & $6.7 \times 10^{-6}$ & $2.7 \times 10^{-2}$ & $2.2 \times 10^{-1}$ \\
\hline
\end{tabular}
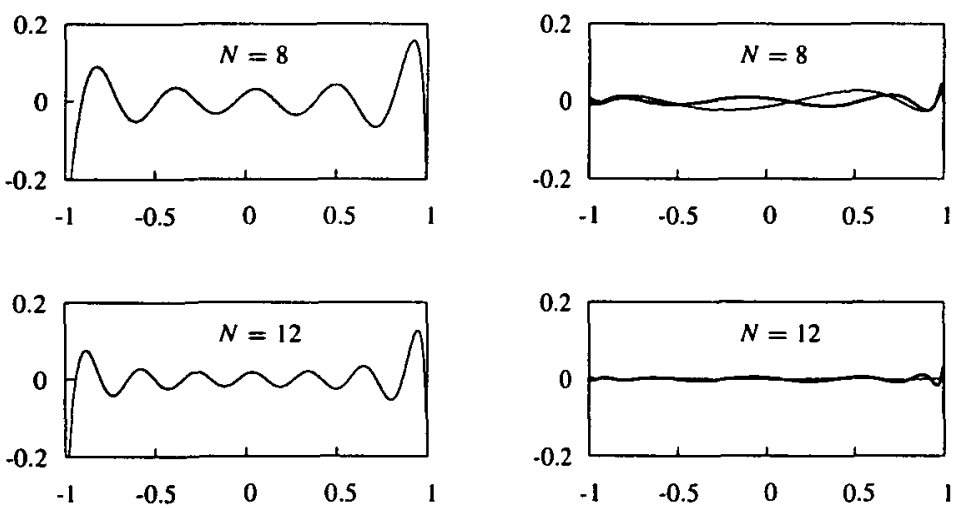

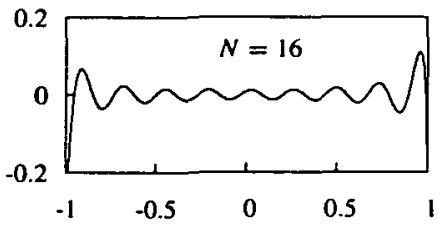

(a)

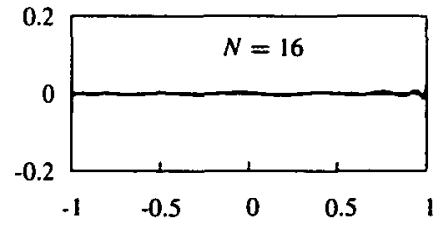

(b)

FIGURE 4. Graphs of the differences $f_{2}-S_{N}^{\mathrm{F}}$ (in (a)) and $f_{2}-S_{N}^{|r|}$ (in (b)). In (b) the thin line and the thick one indicate $S_{N}^{[1 / 4]}$ and $S_{N}^{[1 / 2 \mid}$, respectively. 

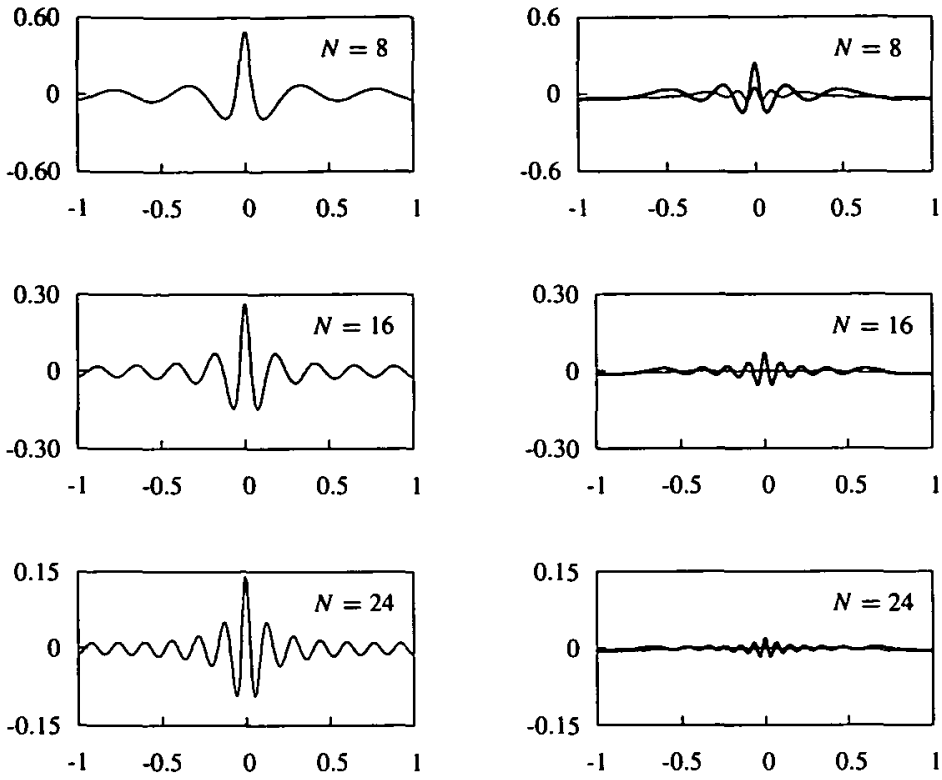

(a)

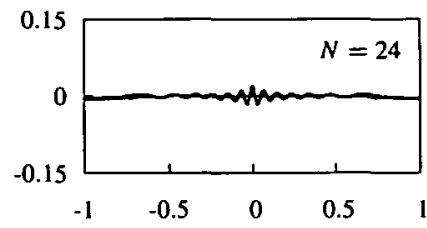

(b)

FIGURE 5. Graphs of the differences $f_{3}-S_{N}^{\mathrm{F}}$ (in (a)) and $f_{3}-S_{N}^{[r]}$ (in (b)). In (b) the thin line and the thick one indicate $S_{N}^{[4]}$ and $S_{N}^{[2]}$, respectively.
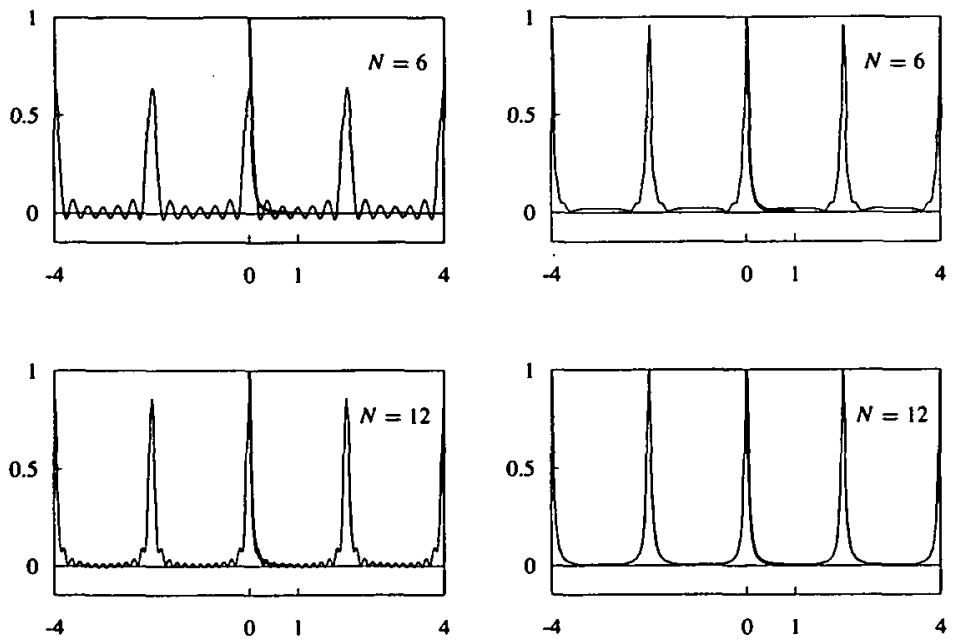

(a)

(b)

FIGURE 6. Periodic even extension of $f_{3}(x), 0 \leq x \leq 1$, by the Fourier series $S_{N}^{\mathrm{F}}$ (in (a)) and the SCS $S_{N}^{(4)}$ (in (b)). The thick line indicates $f_{3}(x)$. 
TABLE 4. Numerical results of $E_{N} f_{3},\left(D_{N} f_{3}\right)(0)$ and $\left(D_{N} f_{3}\right)(1)$ for the SCS and the Fourier series.

\begin{tabular}{ccccc}
\hline & & \multicolumn{2}{c}{ SCS } & Fourier series \\
\cline { 3 - 4 } Errors & $N$ & $S_{N}^{[4]}$ & $S_{N}^{[2]}$ & $S_{N}^{f}=S_{N}^{[1]}$ \\
\hline \multirow{6}{*}{$E_{N} f_{3}$} & 16 & $6.2 \times 10^{-2}$ & $7.6 \times 10^{-2}$ & $1.4 \times 10^{-1}$ \\
& 24 & $4.2 \times 10^{-3}$ & $2.1 \times 10^{-2}$ & $7.4 \times 10^{-2}$ \\
& 32 & $3.5 \times 10^{-3}$ & $1.8 \times 10^{-3}$ & $3.9 \times 10^{-2}$ \\
& 40 & $3.1 \times 10^{-3}$ & $6.1 \times 10^{-4}$ & $1.1 \times 10^{-2}$ \\
& 8 & $5.8 \times 10^{-2}$ & $2.4 \times 10^{-1}$ & $4.9 \times 10^{-1}$ \\
& 16 & $4.7 \times 10^{-3}$ & $6.9 \times 10^{-2}$ & $2.6 \times 10^{-1}$ \\
$\left(D_{N} f_{3}\right)(0)$ & 24 & $4.6 \times 10^{-4}$ & $2.0 \times 10^{-2}$ & $1.4 \times 10^{-1}$ \\
& 32 & $9.8 \times 10^{-5}$ & $5.6 \times 10^{-3}$ & $7.5 \times 10^{-2}$ \\
& 40 & $5.2 \times 10^{-5}$ & $1.6 \times 10^{-3}$ & $4.0 \times 10^{-2}$ \\
& 8 & $2.8 \times 10^{-2}$ & $4.0 \times 10^{-2}$ & $3.9 \times 10^{-2}$ \\
& 16 & $8.6 \times 10^{-3}$ & $1.2 \times 10^{-2}$ & $2.1 \times 10^{-2}$ \\
$\left(D_{N} f_{3}\right)(1)$ & 24 & $6.1 \times 10^{-3}$ & $4.1 \times 10^{-3}$ & $1.1 \times 10^{-2}$ \\
& 32 & $5.3 \times 10^{-3}$ & $1.8 \times 10^{-3}$ & $5.9 \times 10^{-3}$ \\
& 40 & $4.8 \times 10^{-3}$ & $1.1 \times 10^{-3}$ & $3.2 \times 10^{-3}$ \\
\hline
\end{tabular}

large $(N \leq 16)$, approximation by the SCS $S_{N}^{|r|}$ with $r=1 / 4$ or $r=1 / 2$ is very satisfactory compared to the Fourier series approximation. Further improvement can also be obtained by taking a smaller value of $r<1 / 4$ so long as $N$ is modestly large.

EXAMPLE 6.3. (A rapidly decreasing even function.) Consider

$$
f_{3}(x)=\frac{1}{1+400 x^{2}}
$$

Referring to the proof of Theorem 3.3, we can note that since the derivative $f_{3}^{\prime}(x)$ is very small near $x \pm 1$, the SCS $S_{N}^{[r]}$ with somewhat large $r>1$ is also uniformly convergent on the interval $[-1+\epsilon, 1-\epsilon]$ for sufficiently small $\epsilon>0$. In fact, by the numerical fulfillment, we have found that approximation by the $\operatorname{SCS} S_{N}^{[r]}$ with $r>1$ rather than $0<r \leq 1$ gives a better result.

Table 4 includes the errors $E_{N} f_{3}$ and the values of a function $\left(D_{N} f_{3}\right)(x)$ at $x=0$ and $x=1$. Therein $r=2$ and $r=4$ are employed in $S_{N}^{[r]}$, and it can be noted that since $f_{3}$ is an even function, $S_{N}^{\mathrm{F}}=S_{N}^{[1]}$. One can see that $S_{N}^{[r]}$ results in better approximation, in particular, at the central point $x=0$ compared with $S_{N}^{\mathrm{F}}$. Figure 5 shows the graphs of $f_{3}-S_{N}^{[r]}$, with $r=2$ and 4 , in comparison with those of $f_{3}-S_{N}^{F}$ for $N=8,16$ and 24 .

On the other hand, assuming that $f_{3}(x)$ is defined on the half-interval $[0,1]$, the 
periodic even extension by the $\operatorname{SCS} S_{6}^{[4]}$ and $S_{12}^{[4]}$ using the periodic sigmoidal transformation $\gamma_{4}^{*}$ in (5.2) is shown in Figure 6.

\section{Acknowledgements}

The author would like to express his sincere gratitude to the Advanced Computational Modelling Centre (ACMC) at the University of Queensland where the author worked as a visiting scholar for a year. In particular, the author is heartily thankful to Professor Kevin Burrage, Professor John Belward and Mrs Marian Lipman for their favour and kind assistance during the author's visit to the ACMC.

This work was also supported by the Korea Research Foundation (Grant KRF2004-013-C00003).

\section{References}

[1] U. J. Choi, S. W. Kim and B. I. Yun, "Improvement of the asymptotic behavior of the EulerMaclaurin formula for Cauchy principal value and Hadamard finite-part integrals", Int. J. Numer. Meth. Engng. 61 (2004) 496-513.

[2] D. Elliott, "The Euler-Maclaurin formula revisited", J. Austral. Math. Soc. Ser. B 40(E) (1998) E27-E76.

[3] D. Elliott, "Sigmoidal transformations and the trapezoidal rule", J. Austral. Math. Soc. Ser. B 40(E) (1998) E77-E137.

[4] D. Elliott and E. Venturino, "Sigmoidal transformations and the Euler-Maclaurin expansion for evaluating certain Hadamard finite-part integrals", Numer. Math. 77 (1997) 453-465.

[5] G. B. Folland, Fourier Analysis and Its Applications (Wadsworth \& Brooks/Cole, 1992).

[6] I. S. Gradshteyn and I. M. Ryzhik, Table of Integrals, Series, and Products, 6th ed. (Academic Press, San Diego, CA, 2000).

[7] A. J. Jerri, The Gibbs Phenomenon in Fourier Analysis, Splines and Wavelet Approximations (Kluwer Academic Publisher, Dordrecht, 1998).

[8] P. R. Johnston, "Application of sigmoidal transformations to weakly singular and near singular boundary element integrals", Int. J. Numer. Meth. Engng. 45 (1999) 1333-1348.

[9] A. Pinkus and S. Zafrany, Fourier Analysis and Integral Transforms (Cambridge University Press, Cambridge, 1997).

[10] S. Prössdorf and A. Rathsfeld, "On an integral equation of the first kind arising from a cruciform crack problem", in Integral Equations and Inverse Problems (eds. V. Petkov and R. Lazarov), (Longman, Harlow, 1991) 210-219.

[11] A. Sidi, "A new variable transformation for numerical integration", in Numerical Integration IV, ISNM Vol. 112 (eds. H. Brass and G. Hämmerlin), (Birkhaüser, Basel, 1993) 359-373.

[12] B. I. Yun, "An extended sigmoidal transformation technique for evaluating weakly singular integrals without splitting the integration interval", SIAM J. Sci. Comput. 25 (2003) 284-301.

[13] B. I. Yun, "A generalized non-linear transformation for evaluating singular integrals", Int. J. Numer. Meth. Engng. 65 (2006) 1947-1969.

[14] B. I. Yun and P. Kim, "A new sigmoidal transformation for weakly singular integrals in the boundary element method", SIAM J. Sci. Comput. 24 (2003) 1203-1217. 